\title{
Parenting Stress as a Mediator between Mental Health Consultation and Children's Behavior
}

\author{
Stacy L. Bender ${ }^{1}$, John S. Carlson ${ }^{2}$, Laurie Van Egeren ${ }^{2}$, Holly Brophy-Herb ${ }^{2} \&$ Rosalind Kirk ${ }^{3}$ \\ ${ }^{1}$ Alfred University, New York, USA \\ ${ }^{2}$ Michigan State University, Michigan, USA \\ ${ }^{3}$ University of Michigan, Michigan, USA \\ Correspondence: Stacy L. Bender, College of Professional Studies, Alfred University, Alfred, NY, 14802, USA. \\ Tel: 1-607-871-2649. E-mail: bendersl@alfred.edu
}

Received: November 22, 2016

doi:10.5539/jedp.v7n1p72
Accepted: December 6, 2016 Online Published: December 8, 2016

URL: http://dx.doi.org/10.5539/jedp.v7n1p72

\begin{abstract}
Early Childhood Mental Health Consultation (ECMC) focuses on enhancing adults' (e.g., parents) skills and abilities in order to improve children's behavior. Limited research has examined parenting factors as mechanisms of change, which is important given the bidirectional nature of parent-child interactions. Parenting stress and its influence on children's behavioral outcomes (behavior problems and protective factors) were investigated following the implementation of an Early Childhood Mental Health Consultation (ECMHC) program. Participants included parents that participated in the ECMHC program $(n=247)$ and a comparison group $(\mathrm{n}=72)$ in the Midwest. Overall, parents in the ECMHC group experienced fewer dysfunctional parent-child interactions and less distress. Results indicated that parent-child dysfunctional interactions mediated the relationship between ECMHC and children's behavior problems $(\mathrm{CI}=.001, .038)$ and protective factors $(\mathrm{CI}$ $=-.061,-.001)$. Parental distress did not mediate the relationship between ECMHC and children's behavior problems $(\mathrm{CI}=-.001, .016)$ or protective factors $(\mathrm{CI}=-.020, .001)$. Understanding the influence of stress and parent-child interactions is beneficial as these may be malleable and responsive to change if targeted in intervention. Examining mechanisms of change related to parents will allow for refinement of services and improved behavioral outcomes for children.
\end{abstract}

Keywords: behavior concerns, early childhood mental health consultation, parenting stress, protective factors

\section{Introduction}

Behavior problems exhibited by young children often lead to difficulties and dysfunction in adolescence and adulthood (Fergusson, Horwood, \& Ridder, 2005). To avoid the negative long-term consequences of behavior problems, the implementation of prevention and early intervention services such as Early Childhood Mental Health Consultation (ECMHC) have been on the rise (Green, Everhart, Gordon, \& Gettman, 2006). ECMHC is an indirect problem-solving approach to intervening with young children (ages birth to 6 years), their families, and other adult care providers (Cohen \& Kaufman, 2000). This problem-solving approach is developed and implemented collaboratively by a consultant, individuals with other areas of expertise, such as a childcare provider or preschool teacher, and the caregivers of the target child. ECMHC targets adults by enhancing their skills and abilities to better prevent, identify, and treat mental health problems as a way to change children's behavior. ECMHC directly focuses on adults' behavior, which is proposed to lead to indirect effects on children's behavior. The most common type of consultation, child- or family-centered consultation, focuses on impacting childcare factors and family factors that may be contributing to or maintaining the child's challenging behavior. This is advantageous in that it considers multiple ecologies affecting a child by incorporating important adults in a collaborative problem-solving approach.

Research on the effectiveness of ECMHC provides evidence for improving young children's social, emotional, and behavioral functioning. ECMHC has been linked to less challenging behavior (e.g., disruptive behavior; Raver et al., 2009), more positive behaviors (e.g., social skills; Perry, Dunne, McFadden, \& Campbell, 2008), and lower rates of expulsion in at-risk preschool populations (e.g., Upshur, Wenz-Gross, \& Reed, 2009). Given 
the emphasis of changing adult-child interactions in this intervention, it is not only important to investigate outcomes of this approach and if children improve, but also how the intervention helps children improve. Research has hypothesized how changes in childcare providers and teachers help children, such as an improved provider-child relationship and increased teacher efficacy (e.g., Brennan, Bradley, Allen, \& Perry, 2008; Johnston \& Brinamen, 2012), but even this literature base is lacking empirical support and authors have argued for a better understanding of how change occurs in ECMHC. Even more limited is research devoted to understanding how parent changes resulting from ECMHC lead to improved child behavior outcomes. Given that parents spend the most time with their young children and the significant influence parental behavior has on child behavior, examining mechanisms of change related to parents is an essential next step in further establishing ECMHC as an evidence-based practice.

Parents' behavior significantly influences child behavior through parent-child interactions (Patterson \& Reid, 1970). Parenting stress, or the aversive psychological reaction to the demands of being a parent (Abidin, 1992), is one factor that may influence parenting behavior. Parents with elevated parenting stress may be more likely to engage in aversive parenting behaviors. Frequent negative parent-child interactions may result in elevated parenting stress over time. Parents who are highly stressed may be more likely to give in to their child's demands to avoid putting in the effort necessary to effectively manage challenging behavior. Elevated levels of parenting stress are associated with behavior problems and conversely, more behavior problems have been linked to elevated parenting stress (e.g., Barry, Dunlap, Cotton, Lochman, \& Wells, 2005). The intervention literature also suggests relations between parenting stress and child behavior. For example, in studies targeting children's behavior problems, parenting stress is reduced, and when parenting stress is targeted in treatment, children exhibit fewer behavior problems (e.g., Feinfield \& Baker, 2004; Kazdin \& Whitley, 2003).

Given this, it could be the case that if stress is reduced for parents following ECMHC, child behavior problems may also decrease. One component of ECMHC that may affect parents' stress levels is parenting education and skills. Lack of parenting competence, fewer emotional resources, and fewer instrumental resources contribute to parenting stress (Deater-Deckard, 1998). Improving parenting stress levels through ECMHC may enhance parents' perception of available resources, change attitudes toward parenting, and build more effective parenting skills. Reducing parenting stress through ECMHC may lead to positive parent-child interactions and ultimately help to improve children's behavior.

The current study was part of a larger study of ECMHC and extends the findings of the report from that evaluation (Van Egeren et al., 2011). While the large-scale, mixed-method evaluation furthered the research on ECMHC and examined many variables, rationale for why improvements and potential mediators between ECMHC and child outcomes were not investigated. The purpose of the current study was to evaluate parenting stress and its influence on children's behavioral outcomes (i.e., behavior problems and protective factors) following the implementation of an ECMHC program. Research has suggested that higher levels of parenting stress is related to more behavior problems exhibited by children, and that behavior problems and parenting stress often predict the presence and level of the other (e.g., Barry et al., 2005). Additionally, the mediating role of parenting stress in improving children's behavioral outcomes following intervention is an important area of investigation (Feinfield \& Baker, 2004). In this study, the first hypothesis was that parenting stress would mediate the relation between ECMHC and child behavior problems. More specifically, we purported that if parenting stress was high after ECMHC, more behavior problems would be reported, and if parenting stress was rated as being low after ECMHC, less behavior problems would be found after the intervention.

A paucity of research has been dedicated to examining whether less parenting stress may be related to children displaying more protective factors. This includes behaviors such as self-control, initiative, attachment, and the ability to communicate effectively (LeBuffe \& Naglieri, 1999). Protective factors promote resiliency and moderate or buffer the negative effects of stress (Rutter, 1987). Previous studies indicate that higher levels of parenting stress are related to lower levels of children's social competence (e.g., Anthony et al., 2005; Bender \& Carlson, 2013). Given this previous finding, the second hypothesis was that parenting stress would mediate the relation between the ECMHC and children's protective factors. Specifically, we predicted that less parenting stress would be associated with more protective factors and more parenting stress would be associated with fewer protective factors following ECMHC. 


\section{Method}

\subsection{Participant Characteristics}

Three hundred nineteen parents of children between the ages of 6 and 72 months participated. Two groups of participants were included in the study: the ECMHC group $(\mathrm{N}=247)$ and a comparison group $(\mathrm{N}=72)$. The ECMHC group included parents who participated in the Child Care Expulsion Prevention (CCEP) Program, a state-funded initiative in the Midwest between the years of 2007 to 2010. The comparison group included parents that did not participate in the CCEP program and resided in counties in the state that did not have access to ECMHC (i.e., CCEP) services. Both groups involved children who were demonstrating behavioral challenges impacting their functioning in the childcare center and/or at home.

\subsubsection{CCEP Group}

Children attending childcare programs in the counties that offered ECMHC services were referred to the CCEP program by their childcare provider or parent because of the child's behavioral difficulties exhibited in the childcare or home setting. Children were referred for several reasons, including problems related to aggression, developmental delays, behavioral regulation, physical difficulties, sensory integration, or other types of externalizing behavior. After consultants made initial contact with the provider and family, consultants obtained consent for CCEP services as well as consent for the data to be sent to independent evaluators at the university. When university consent was given, the parents completed several questionnaires related to their child's behavior. Following the conclusion of services parents completed the same questionnaires, along with a survey assessing their satisfaction with the consultation process and effectiveness. After the pre and post questionnaires were completed, CCEP consultants sent the data to the researchers evaluating the CCEP program.

CCEP was flexible in its approach given the diverse needs and challenges of the child and complexity and severity of the problem. Number of visits and duration of services were not uniform given that they were tailored to each particular child, parent, and provider. However, on average, consultants spent one to three hours per week with an individual child/family and provider. CCEP services typically lasted three to six months for most children.

Consultants served children and families from 31 counties throughout the state. The age of children in the ECMHC group ranged from 12 to 72 months ( $\mathrm{M}=44$ months; $\mathrm{SD}=12.5$ months), with $76 \%$ being male. Approximately $81 \%$ of children were identified as Caucasian. The majority of children (92\%) exhibited multiple behavior problems. Children were most frequently referred to ECMHC for problems related to aggression, developmental delays, and self-regulation, as reported by their parents. Of the children included in the sample, $28 \%$ exhibited difficulties related to developmental delays (e.g., exhibiting high activity levels), $22 \%$ presented with some type of aggression problem (e.g., hitting, verbal aggression), and $21 \%$ had regulatory problems (e.g., inability to self-regulate, demanding behavior). The majority of parents in the ECMHC group were female ( $\mathrm{N}=$ $237 ; 96 \%)$ with an age range of 20 to 59 years ( $\mathrm{M}=33$ years; $\mathrm{SD}=6.9$ years). The majority of parents identified as Caucasian (84\%). Parents reported their income in the past 12 months. Sixteen percent reported less than $\$ 14,999,49 \%$ fell between $\$ 15,000$ and $\$ 54,999,25 \%$ fell between $\$ 55,000$ and $\$ 99,999$, and $10 \%$ reported more than $\$ 100,000$.

\subsubsection{Comparison Group}

The evaluators recruited families for the comparison group by sending advertisements to licensed family daycare centers and childcare centers in counties that did not offer ECMHC services. Recruitment flyers were also posted in the community (e.g., grocery stores, doctor's offices), in early childhood newsletters, and websites. Interested parents who inquired about participation were asked a series of questions over the phone to assess child behavior and determine eligibility. Questions assessed child behavior to determine whether behavior problems were comparable to the behavior problems exhibited by children referred to the CCEP program. Exclusions from participation were made when ratings of child behavior problems fell below a pre-defined cutoff score, if the child attended a publically funded preschool, or if their child attended Head Start. Eligible parents were provided information about the study and interested parents provided verbal consent over the phone. Phone interviews were scheduled within one week and took 30 to 45 minutes. Questionnaires assessed the child's behavior and parent characteristics such as parenting stress and empowerment. In rare cases, parents were sent a copy of the questionnaires in the mail to complete and mail back to the evaluators. Six months later, parents were contacted again to complete the second phone interview. The same questionnaires were administered as the initial interview. 
The age of children ranged from 6 to 64 months $(M=37$ months; $S D=13.8$ months), with $64 \%$ being male. Approximately $86 \%$ of children were identified as Caucasian. Similar to the CCEP group, the majority of children exhibited multiple behavior problems (84\%). Parents most frequently reported behavior problems related to developmental delays $(40 \%)$, aggression $(33 \%)$ and self-regulation $(21 \%)$. The majority of parents in the comparison group were female $(\mathrm{N}=68 ; 94 \%)$ with an age range of 19 to 46 years $(\mathrm{M}=31$ years; $\mathrm{SD}=5.9$ years). Parents reported their income in the past 12 months. Twenty-one percent reported less than $\$ 14,999,42 \%$ fell between $\$ 15,000$ and $\$ 54,999,27 \%$ fell between $\$ 55,000$ and $\$ 99,999$, and $10 \%$ reported more than $\$ 100,000$.

\subsection{Procedure}

\subsubsection{Description of the Child Care Expulsion Prevention (CCEP) Program}

CCEP was an ECMHC program funded by the Department of Community Health (MDCH) in the Midwest where consultants worked collaboratively with child care providers, parents, and administrators to promote healthy social-emotional development for young children and prevent expulsion (Carlson et al., 2012). The CCEP program served children age birth to five years in 31 of 83 counties. The fundamental approach to serving young children is relationship-based practice, where consultants facilitate, and nurture adult-child interactions. Consultants also equipped these adults with knowledge and skills that improved their interactions with the child.

The CCEP program provided family/child-centered consultation and programmatic consultation. However the main focus was child/family-centered consultation. Overall, CCEP had all of the components that are considered to be essential in ECMHC including emphasis on a collaborative relationship between the consultant, parent, and provider, utilization of problem-solving and creation of skill-building goals, identification of specific issues to be targeted and addressing issues within a reasonable time frame, and consultants who possess a set of professional skills and interpersonal skills. In addition to meeting this criteria of ECMHC, CCEP had specific steps that were integral to CCEP implementation. These steps include: a) referral, intake, and consent; (b) observation and assessment; (c) meeting with parents and providers to develop a Positive Child Guidance Plan; (d) support to implement the Positive Child Guidance Plan; (e) referrals to outside services as needed; and (f) conclusion of services.

Consultants engaged in several types of services to enhance the attitudes and skills of providers and parents, as well as improve the classroom and home environment. Consultants provided direct and indirect services (Perry et al., 2010) to assess the child's problems. After the child's problem was identified, a plan was constructed to reduce the problem. Consultants often provide leadership to construction of the plan and ensured that the plan was carried out. Indirect services may have included educating providers and parents about a particular topic (e.g., child development and behavior), coaching and modeling of positive adult behavior and discipline strategies, conducting in-service or trainings, and consultation regarding adjustments to the classroom or home environment (e.g., routines, structure, environment layout). Consultants made referrals for the child and/or family to other professionals or agencies if their needs exceeded the resources or expertise of the consultant and ECMHC program. Resources were also provided to families and childcare centers, such as books and toys.

According to a review of ECMHC and its impact on children's behavioral outcomes, Perry and colleagues (2010) highlight three different approaches to ECMHC service delivery within the literature. One of the approaches, individualized consultation services, which is defined as consultation that is tailored to the needs of the child, family, provider, or program, is the type of consultation delivery model used in CCEP. Several evidence-based practices are promoted within the model. This includes the use of valid and reliable assessment tools, as well as the use of effective teaching and scaffolding strategies, such as coaching, modeling, and immediate feedback. Implementation of this approach has been suggested to be a promising way to reduce young children's behavior problems.

Qualified consultants working within community agencies provided CCEP services. Qualifications of CCEP consultants included possessing a) a master's degree in social work, psychology, or related field; b) a license or being license-eligible; and c) a Level II-Infant Mental Health Endorsement for culturally sensitive, relationship-based practice promoting infant mental health, or a graduate certification in infant mental health studies. Consultants were supervised by agency personnel and were required to participate in ongoing reflective supervision. MDCH Technical Assistance (TA) staff also provided support to consultants in various ways such as specialized training, individualized supervision, quarterly meetings, resources, and an email support group. 


\subsubsection{Integrity and Fidelity of CCEP Implementation}

Several variables were evaluated to determine whether CCEP services were implemented as intended for the participants in the current study. The variables were selected based on the larger study of ECMHC Evaluation Report (Van Egeren et al., 2011) and suggestions from the literature (e.g., Sheridan et al., 2009). Completed intake forms and evidence of a Positive Guidance Plan were indicators of high implementation fidelity. These permanent products are also supported in the literature as evidence of implementation fidelity (Sheridan, Swanger-Gagne, Welch, Kwon, \& Garbacz, 2009).

The percentage of participants that completed the intake form in the current study's sample was $100 \%$. Additionally, a Positive Guidance Plan was constructed for $93 \%$ of the children in the current study's sample. Observation and assessment was completed for $100 \%$ of children and $100 \%$ of consultants supported the implementation of the Positive Child Guidance Plan by providing a wide range of services that varied in duration and intensity. As mentioned above, consultants spent an average of one to three hours per week with an individual child/family and provider, with program services typically lasted three to six months for most children.

Self-reported perceptions of consultation services were considered another way of evaluating the fidelity of service implementation (Sheridan et al., 2009). Parents rated their satisfaction with the consultant they worked with and CCEP services. This self-reported information is a form of adherence, as perceived by those participating in CCEP, and yields an estimate of consultees' adherence to or compliance with intervention implementation. The parent satisfaction survey measured how helpful parents found their consultant to be and how willing they would be to request services from their consultant in the future, even if other consultants were available. This survey consisted of the Consultant Evaluation Form (CEF), as well as additional items that assessed parents' perceptions of consultation. In addition to this measure evaluating parents' perception of satisfaction, it was also an indirect way of determining how successful the consultant was at building a relationship with the parent. As indicated by ECMHC literature as well as CCEP cornerstones, relationship building was an important component of services. The parent satisfaction survey was an optional questionnaire that parents completed after CCEP services and mailed to the evaluators in a paid postage, addressed envelope. Given that this measure was optional, not all parents completed the measure. For the current study sample, two-thirds of parents completed and mailed the questionnaire back to the evaluators.

In regards to overall parent satisfaction, scores were generally high, indicating high levels of satisfaction. All questions were rated on a scale of 1 (low, or strong disagreement) to 7 (high, or strong agreement). The CEF consisted of 12 questions with a possible overall score range of 12 to 84. The mean CEF score for the current sample was 76.2 (item mean: 6.4). The mean for individual items that assessed overall perception of consultation (mean $=6.4)$, overall perception of change in competence (mean $=6.0)$, perception of behavior improvement in child $($ mean $=6.0$ ), and time of effectiveness ( incorporated into the satisfaction survey. One subscale assessed parents' acceptability of CCEP services. The possible overall score range was 9 to 63 . The mean score for the current sample was 55.5 (item mean $=6.2$ ). The second subscale assessed parents' perception of effectiveness of CCEP services. The possible overall score range for this subscale was 4 to 28 . The mean score for the current sample was 22.5 (item mean: 5.6).

\subsection{Measures}

\subsubsection{Demographic Information Questionnaire}

The CCEP Intake Form was a questionnaire used to collect descriptive information from parents about their child and family. Child age, gender, race, behavior problems exhibited, and number of previous expulsions was gathered. Family information such as household income, primary language spoken in home, and services received (e.g., food assistance, medical assistance) was also gathered. During the second year of data collection, the intake form was revised to include additional demographic information. Significant missing demographic data resulted from this change.

\subsubsection{Parenting Stress Index-Short Form (Adapted)}

The Parenting Stress Index-Short Form (PSI-SF $3^{\text {rd }}$ Ed.; Abidin, 1995) is a derivative of the Parenting Stress Index (PSI $3^{\text {rd }}$ Ed.; Abidin, 1995). The 36-item PSI-SF is an early identification assessment of parenting and family characteristics that fail to promote normal development and functioning in children, children with behavioral and emotional problems, and parents who are considered at-risk for dysfunctional parenting. This study used the Parent-Child Dysfunctional Interaction and Parental Distress subscales. The Parent-Child 
Dysfunctional Interaction subscale evaluates the parent and child's interactions and measures parents' perceptions that the child does not meet expectations and interactions with the child are not reinforcing. The Parental Distress subscale measures parents' perceptions of child-rearing competence, social support, and stresses associated with the restrictions placed on other life roles. Psychometric research with low-income population supports the use of the PSI-SF (Reitman, Currier, \& Stickle, 2002). The PSI-SF predicts observed parenting behavior as well as children's current and future behavioral and emotional adjustment (Abidin, 1995). Abidin (1995) reported reliability alpha coefficients of 0.80 for Parent-Child Dysfunctional Interaction and 0.87 for Parental Distress subscales.

\subsubsection{Devereux Early Childhood Assessment (DECA)}

The DECA is a nationally, norm-referenced, strength-based assessment tool that evaluates protective and risk factors in preschool children (LeBuffe \& Naglieri, 1999). Three versions of the DECA were available and completed by parents based on the age of the child. The DECA-Infant (1 to 18 months) consists of 33 items that assess for Initiative and Attachment. The DECA-Toddler (18 to 36 months) includes 36 items that assess for Initiative, Attachment, and Self-Regulation. The subscales are combined to create a Total Protective Factor (TPF) score. The DECA-Preschool (2 to 5 years) consists of 37 items; 10 items assess for Behavior Concerns (BC) and 27 items assess TPF. This score is compromised of 3 subscales including Initiative, Self-Control, and Attachment. Each scale has an average $t$-score of 50, with a standard deviation of 10. On all DECA forms, children can receive ratings of Strength $(t \geq 60)$, Typical $(40 \leq t \leq 60)$, or Concern $(t \leq 40)$ on the TPF scale and TPF subscales. On the BC scale, children can also receive ratings of Strength $(t<40)$, Typical $(40 \leq t \leq 60)$, or Concern $(t \geq 60)$. Internal reliability alpha coefficients for the scales based on parent-ratings are: .84 (Initiative), .86 (Self-control), .76 (Attachment), .91 (Total Protective Factors), and .71 (Behavior Concerns) (Lebuffe \& Naglieri, 1999). The standardization sample for the DECA-Infant and DECA-Toddler consisted of 2,183 children and the standardization sample for the DECA-Preschool consisted of 2,000 preschool children, who accurately reflected the diversity of preschool children in the United States. Supportive evidence also exists for use of the DECA with low-income early childhood populations exhibiting behavior problems (Brinkman et al., 2007).

\subsection{Data Analysis}

To investigate the mediating relationships, multiple regression analysis was used to determine whether the ECMHC condition (ECMHC vs. comparison group) and parenting stress significantly contributed to lower ratings of behavior problems. The bootstrap method, a type of mediation analysis that has growing support for testing indirect effects (Hayes, 2009; Preacher, Rucker, \& Hayes, 2007; Shrout \& Bolger, 2002), was performed. This assessed whether parenting stress mediated the relation between the ECMHC condition and behavioral outcomes. The Statistical Package for the Social Sciences (SPSS) was used to conduct all of the analyses. A macro for the bootstrap analysis was downloaded from http://www.afhayes.com/spss-sas-and-mplus-macros-and-code.html and was also conducted in SPSS.

\section{Results}

\subsection{Correlation Analyses and Analyses of Variance}

Pearson correlations were conducted for variables included in the study. Correlation matrices for the ECMHC group (pre and post) and the comparison group (time 1 and time 2) can be found in Table 1. For the ECMHC group, at both time 1 and time 2, the Parental Distress and Parent-Child Dysfunctional Interaction subscales had a medium positive correlation with Behavior Concerns and a medium negative correlation with Total Protective Factors. When parents rated more Parental Distress and Parent-Child Dysfunctional Interactions, they also rated their children as exhibiting more behavior problems and fewer protective factors. For the comparison group at time 1, similar significant correlations were found between variables; however, one difference emerged. Parental Distress and Behavior Concerns were not significantly correlated, whereas Parental Distress and Total Protective Factors were negatively correlated. This suggests that higher ratings of Parental Distress were not necessarily related to more behavior problems but were related to fewer protective factors for children within the comparison group. 
Table 1. Pearson correlations among study variables for CCEP and comparison group

\begin{tabular}{|c|c|c|c|c|c|c|c|c|c|c|c|c|c|c|c|c|}
\hline $\begin{array}{l}\text { ECMHC Group } \\
\text { Variable (Pre) }\end{array}$ & 1 & 2 & 3 & 4 & 5 & 6 & 7 & 8 & 9 & 10 & 11 & 12 & 13 & 14 & 15 & 16 \\
\hline 1. Parental Distress & --- & $.59^{* *}$ & $.35^{* *}$ & $-.32^{* *}$ & & & & & & & & & & & & \\
\hline \multicolumn{17}{|l|}{ Interaction } \\
\hline 3. Child Behavior Concerns & & & --- & $-.51 * *$ & & & & & & & & & & & & \\
\hline 4. Child Total Protective & & & & -- & & & & & & & & & & & & \\
\hline \multicolumn{17}{|l|}{ Factors } \\
\hline \multicolumn{17}{|l|}{ ECMHC Group } \\
\hline \multicolumn{17}{|l|}{ Variable (Post) } \\
\hline 5. Parental Distress & & & & & --- & $.59^{* *}$ & $.30^{* *}$ & $-.33 * *$ & & & & & & & & \\
\hline 6. Parent-Child Dysfunctional & & & & & & --- & $.31^{* *}$ & $-.45^{* *}$ & & & & & & & & \\
\hline \multicolumn{17}{|l|}{ Interaction } \\
\hline 7. Child Behavior Concerns & & & & & & & --- & $-.48^{* *}$ & & & & & & & & \\
\hline 8. Child Total Protective & & & & & & & & -- & & & & & & & & \\
\hline \multicolumn{17}{|l|}{ Factors } \\
\hline \multicolumn{17}{|l|}{ Comparison Group } \\
\hline \multicolumn{17}{|l|}{ Variable (Pre) } \\
\hline 9. Parental Distress & & & & & & & & & -- & $.62 * *$ & .09 & $-.31 * *$ & & & & \\
\hline 10. Parent-Child Dysfunctional & & & & & & & & & & --- & $.25^{* *}$ & $-.49 * *$ & & & & \\
\hline \multicolumn{17}{|l|}{ Interaction } \\
\hline 11.Child Behavior Concerns & & & & & & & & & & & --- & $-.57 * *$ & & & & \\
\hline 12. Child Total Protective & & & & & & & & & & & & -- & & & & \\
\hline \multicolumn{17}{|l|}{ Factors } \\
\hline \multicolumn{17}{|l|}{ Comparison Group } \\
\hline \multicolumn{17}{|l|}{ Variable (Post) } \\
\hline 13. Parental Distress & & & & & & & & & & & & & --- & $.52^{* *}$ & $.43^{* *}$ & $-.33^{* *}$ \\
\hline 14. Parent-Child Dysfunctional & & & & & & & & & & & & & & --- & $.37^{* *}$ & $-.43^{* *}$ \\
\hline \multicolumn{17}{|l|}{ Interaction } \\
\hline 15. Child Behavior Concerns & & & & & & & & & & & & & & & --- & $-.10^{* *}$ \\
\hline 16. Child Protective Factors & & & & & & & & & & & & & & & & -- \\
\hline
\end{tabular}

$* * \mathrm{p} \leq .01$

Additionally, point biserial correlations including the ECMHC and comparison group variables were conducted (Table 2). At pre and post, the ECMHC group had a small negative correlation with the Parental Distress and Parent-Child Dysfunctional Interaction subscales. Parent participation in the treatment group was related to lower ratings of Parental Distress and Parent-Child Dysfunctional Interactions. Interestingly, the ECMHC Group variable was not significantly correlated with child Behavior Concerns or Protective Factors. Unlike parents in the treatment group, children's participation in the treatment group was not related to fewer behavior problems and more protective factors. Parental Distress had a medium positive correlation with Behavior Concerns and medium negative correlation with Protective Factors. Parent-Child Dysfunctional Interaction also had a medium positive correlation with Behavior Problems and medium negative correlation with Protective Factors. Similar to the significant correlations discussed above, this suggests that more elevated ratings of Parental Distress and Parent-Child Dysfunctional Interactions were related to more behavior problems and fewer protective factors. 
Table 2. Point Biserial correlations between treatment and comparison study variables

\begin{tabular}{|c|c|c|c|c|c|c|c|c|c|c|}
\hline Variable (Pre) & 1 & 2 & 3 & 4 & 5 & 6 & 7 & 8 & 9 & 10 \\
\hline 1. Treatment Group (ECMHC vs. Comparison) & --- & $-.13^{*}$ & $-.16^{*}$ & -.06 & -.00 & & & & & \\
\hline 2. Parental Distress & & --- & $.61 * *$ & $.30^{* *}$ & $-.31 * *$ & & & & & \\
\hline 3. Parent-Child Dysfunctional Interaction & & & --- & $.35^{* *}$ & $-.44 * *$ & & & & & \\
\hline 4. Behavior Concerns & & & & --- & $-.52 * *$ & & & & & \\
\hline 5. Total Protective Factors & & & & & --- & & & & & \\
\hline \multicolumn{11}{|l|}{ Variable (Post) } \\
\hline 6. Treatment Group (ECMHC vs. Comparison) & & & & & & --- & $-.11^{*}$ & $-.18 * *$ & -.04 & .10 \\
\hline 7. Parental Distress & & & & & & & --- & $.58 * *$ & $.33^{* *}$ & $-.34 * *$ \\
\hline 8. Parent-Child Dysfunctional Interaction & & & & & & & & --- & $.32 * *$ & $-.45^{* *}$ \\
\hline 9. Behavior Concerns & & & & & & & & & --- & $-.41 * *$ \\
\hline 10. Total Protective Factors & & & & & & & & & & --- \\
\hline
\end{tabular}

Independent samples $t$-test analyses were conducted to determine whether differences at time 1 between the two groups existed in terms of children's behavior and parents' stress. Behavior Concerns scores were not found to be significantly different between ECMHC $(\mathrm{M}=65.09, \mathrm{SD}=7.81)$ and comparison $(\mathrm{M}=66.16, \mathrm{SD}=7.92)$ groups $(t=1.02, \mathrm{df}=317, \mathrm{p}>.05)$. Total Protective Factor scores were not found to be significantly different between ECMHC $(\mathrm{M}=41.04, \mathrm{SD}=9.89)$ and comparison $(\mathrm{M}=41.06, \mathrm{SD}=9.76)$ groups $(t=.014, \mathrm{df}=317$, $\mathrm{p}>.05$ ). Furthermore, scores for Parent-Child Dysfunctional Interaction were not significantly different between ECMHC $(\mathrm{M}=21.05, \mathrm{SD}=6.44)$ and comparison $(\mathrm{M}=23.49, \mathrm{SD}=5.84)$ groups $(t=2.88, \mathrm{df}=317, \mathrm{p}>.05)$. The Parental Distress scores were not significantly different between ECMHC $(\mathrm{M}=25.80, \mathrm{SD}=8.57)$ and comparison $(\mathrm{M}=28.39, \mathrm{SD}=8.21)$ groups $(t=2.27, \mathrm{df}=317, \mathrm{p}>.05)$.

A mixed between-within subjects ANOVA was conducted to determine whether changes in scores were the same over time for the two different groups. Children's Behavior Concerns $(F(1,317)=53.36, p<.01)$ and Total Protective Factors $(\mathrm{F}(1,317)=44.90, \mathrm{p}<.01)$ were significantly different over time for both groups. No significant differences emerged between groups on post scores, suggesting that scores for Behavior Concerns $(\mathrm{F}(1,317)=.35, \mathrm{p}>.05)$ and Total Protective Factors $(\mathrm{F}(1,317)=.99, \mathrm{p}>.05)$ were similar. However, differences emerged for the interaction effects. No significant interaction between group and time were apparent for Behavior Concerns $(\mathrm{F}(1,317)=.06, \mathrm{p}>.05)$, whereas a significant interaction was found for Total Protective Factors $(\mathrm{F}(1,317)=3.89, \mathrm{p}<.05)$.

A mixed between-within subjects ANOVA was conducted for the Parental Distress and Parent-Child Dysfunctional Interaction subscales. Parental Distress $(\mathrm{F}(1,317)=31.81, \mathrm{p}<.01)$ and Parent-Child Dysfunctional Interactions $(\mathrm{F}(1,317)=.02, \mathrm{p}<.01)$ were significantly different over time for both groups. The ECMHC group experienced less distress $(\mathrm{F}(1,317)=5.46, \mathrm{p}<.05)$ and fewer dysfunctional interactions $(\mathrm{F}(1$, $317)=11.33, \mathrm{p}<.01)$ with their children at time 2 . No significant interaction between group and time were apparent for either subscale, suggesting that that change in scores over time for the two different groups were similar.

\subsection{Regression and Mediation Analyses}

Although the preliminary point biserial correlations between the ECMHC Group condition and children's behavioral outcomes (Behavior Concerns, Total Protective Factors) were not significantly correlated, the mediation analysis was still conducted. This was justified by the mediation guidelines set forth by Shrout and Bolger (2002). They recommend moving forward with mediation analyses even if the X (ECMHC Group) $\rightarrow$ Y (children's behavioral outcomes) relation is not significant. They argue that as causal processes become more distal, the effect size may become smaller because it is more likely to be transmitted through additional links in a causal chain, affected by competing causes, or affected by random factors. It may be the case that the more proximal $\mathrm{X} \rightarrow \mathrm{M}$ and $\mathrm{M} \rightarrow \mathrm{Y}$ associations are larger than the distal $\mathrm{X} \rightarrow \mathrm{Y}$ association therefore the test of $\mathrm{X} \rightarrow$ $\mathrm{Y}$ may be more powerful when mediation is taken into account. It is also suggested that the analyses proceed on the basis on the strength of the theoretical argument between $\mathrm{X} \rightarrow \mathrm{Y}$, rather than the statistical test of $\mathrm{X}$ on $\mathrm{Y}$. 
To test mediating relationships, the SPSS macros for mediation (Hayes, 2011; Hayes, 2012; Preacher \& Hayes, 2008) were utilized. The macro gave estimates of the specific indirect effects as well as of the total indirect effect. The current analysis utilized 1,000 bootstrap samples that were created from the original dataset $(\mathrm{N}=319)$ by random sampling with replacement. Ninety-five percent confidence intervals $(95 \% \mathrm{CI})$ were used to evaluate the significance and magnitude of indirect effects estimated through the bootstrap method. Based on Shrout and Bolger's (2002) suggestion, if the $95 \% \mathrm{CI}$ for the estimates of the indirect effects based on these 1,000 indirect effect estimates does not include zero, then it can be concluded that the indirect effect is statistically significant at the .05 level.

The results of the analysis showed that the ECMHC group was associated with a negative direct effect on parental distress $(\beta=-.11, p<.05)$ and parent-child dysfunctional interaction $(\beta=-.18, p<.01)$. Parental Distress $(\beta=.33, p<.001)$ and Parent-Child Dysfunctional Interaction $(\beta=.32, p<.001)$ also had a positive direct effect on children's behavior problems. This suggests that parents in the ECMHC group experienced less parental distress and dysfunctional interactions following ECMHC. Additionally, parents with more stress had children with more behavior problems. No direct effect was found between the Treatment Group and children's behavior problems even after controlling for both Parental Distress $(\beta=-.001, p>.05)$ and Parent-Child Dysfunctional Interaction $(\beta=.02, p>.05)$. Participation in the treatment group did not predict fewer behavior problems for children. These effects were lower after controlling for the parenting stress subscales, compared to without controlling for them.

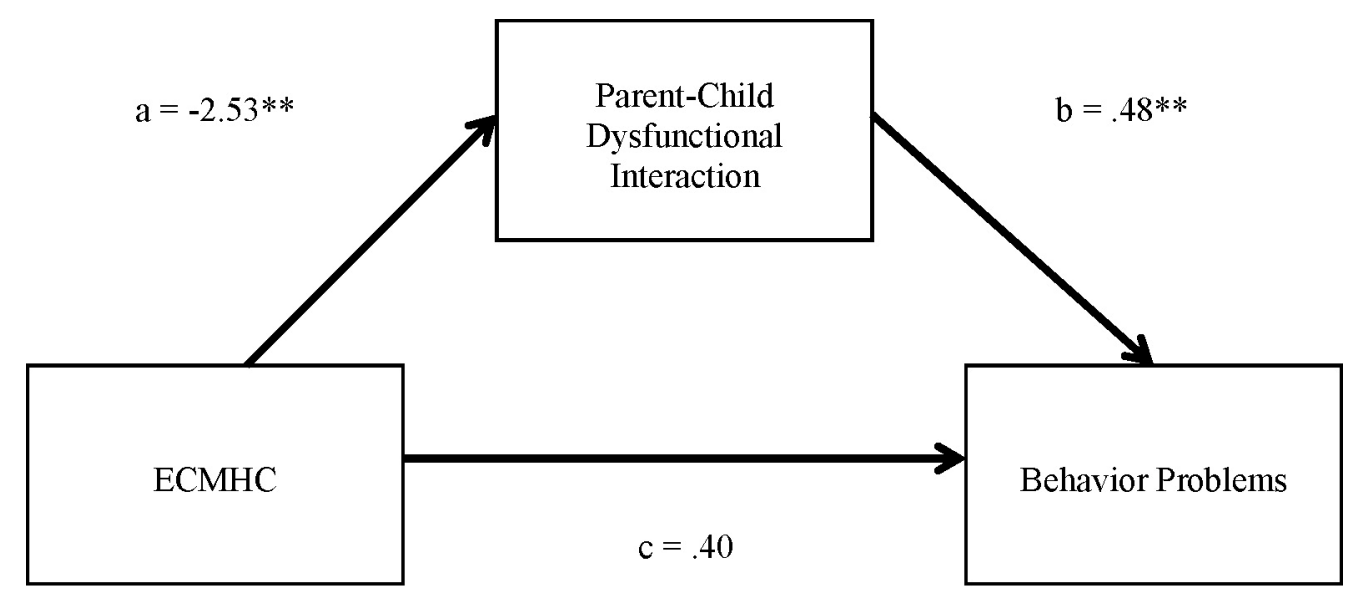

Total Indirect Effect $=-1.21^{*}$

(CI: $-2.03,-.48)$

Figure 1. Significant mediation results for parent-child dysfunctional interaction and children's behavior $\left({ }^{*} \mathrm{p}\right.$

$$
<.05, * * \mathrm{p}<.001)
$$

For the parental distress model, the total indirect effect was not significant $(\mathrm{CI}=-.001,0.16)$. In the Parent-Child Dysfunctional Interaction model, the total indirect effect was significant with the $95 \%$ bootstrap confidence interval excluding zero $(\mathrm{CI}=.001, .038)$ (Table 6). This model suggests that the relationship between the ECMHC group and children's behavior problems was mediated by Parent-Child Dysfunctional Interaction, but not Parental Distress (Figure 1).

In examining the indirect effects on children's total protective factors, the results showed that the ECMHC group had a negative direct effect on Parental Distress $(\beta=-.11, p<.05)$ and Parent-Child Dysfunctional Interaction $(\beta$ $=-.18, p<.01)$. Parental Distress $(\beta=-.34, p<.001)$ and Parent-Child Dysfunctional Interaction $(\beta=-.45, p$ $<.001)$ had a negative direct effect on children's total protective factors. Similar to the previous mediation model, the results suggest that parents in the ECMHC group experienced less parental distress and dysfunctional interactions. Additionally, parents with more stress had children with fewer protective factors. No direct effect was found between the ECMHC Group and children's total protective factors, even after controlling for both Parental Distress $(\beta=.06, p>.05)$ and Parent-Child Dysfunctional Interaction $(\beta=.02, p>.05)$. Participation in 
the ECMHC group did not predict more protective factors for children. These effects were lower after controlling for the parenting stress subscales.

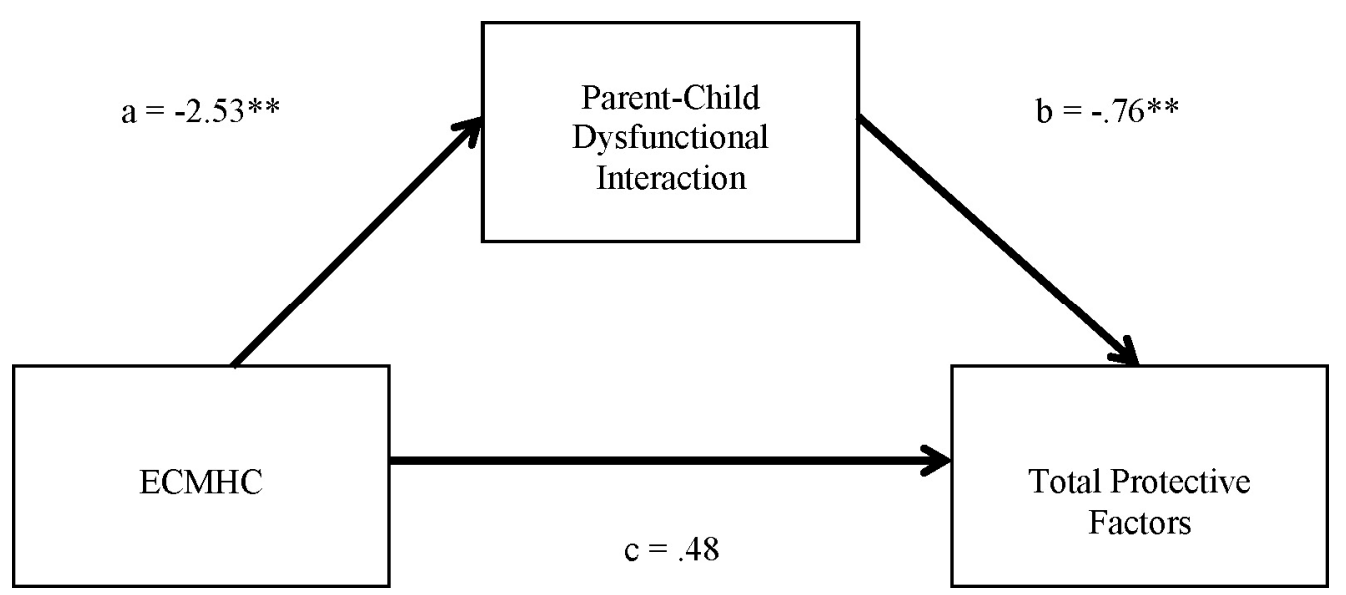

Figure 2. Significant mediation results for parent-child dysfunctional interaction and children's protective factors

$$
\left(* \mathrm{p}<.05,{ }^{* *} \mathrm{p}<.001\right)
$$

For the parental distress model, the total indirect effect was not significant. In the Parent-Child Dysfunctional Interaction model, the total indirect effect was significant with the $95 \%$ bootstrap confidence interval excluding zero $(\mathrm{CI}=-.061,-.001)$ (Table 4). The relationship between the ECMHC group and children's total protective factors was mediated by Parent-Child Dysfunctional Interaction (Figure 2).

\section{Discussion}

This is the first study to appear within the literature examining mediators of ECMHC on child behavior outcomes. Results suggested that parent ratings of dysfunctional interactions with their child played a mediating role in the relationship between ECMHC and children's behavior problems and protective factors. Parent ratings of parental distress were not found to be a mediator between ECMHC and children's behavioral outcomes. Results also suggested that parents in the ECMHC group experienced less distress and fewer dysfunctional parent-child interactions.

In the current study, parents in the ECMHC group who experienced more stress had children with more behavior problems and parents with less stress had children with more protective factors. This is consistent with Feinfield and Baker's (2004) study, which demonstrated that when parents received an intervention component focusing on parent problem solving, greater therapeutic change was evident for the child. Their results indicated that parent factors, such as attitude, stress, and parenting practices played a role in the relationship between the treatment (which consisted of child- and parent-focused components) and children's behavioral outcomes. Their study demonstrated that parents' attitude, measured by negative perceptions of the parent-child relationship and parenting efficacy, and stress, as measured by the Child Stress subscale of the PSI, mediated the effects of intervention on children's behavior problems. When parents experienced fewer negative perceptions about their children and reported less stress caused by their children, children's behavior was likely to improve.

Research has suggested that a contributing factor to parenting stress is parents' perception of access to resources (Deater-Deckard, 1998). Parenting resources include knowledge and competence of parenting tasks. Thus, parents who have less knowledge about child development and child-rearing, lower feelings of parenting competence, fewer emotional resources, and fewer instrumental resources are likely to experience more stress. Given that ECMHC in the current study provided parents with various resources, including knowledge, skills, and support by a consultant, it could be that parents in the ECMHC group experienced a reduction in stress as a result of improved access to practical support, greater understanding of their children's behaviors, and guidance on effective parenting techniques. In turn, reductions in stress may have led to an increased use of positive techniques and helped to improve their child's behavior following ECMHC. Additionally, emotional support provided by the consultant to the parent may also have contributed to stress reduction since research has shown positive links between these factors (Kirk, 2003). 
Parenting stress influences children's behavior problems as well as protective factors. Literature has suggested that parenting stress affects parenting behavior and parents' feelings toward their child (Deater-Deckard, 1998). Therefore, parents with less stress may have more positive feelings toward their children and more positive interactions with their children. Opposite to the coercive parenting cycle (Patterson, 1982), positive interactions toward the child may elicit more positive behaviors from the child, thus resulting in parents' perception of improved behavior. Children may also observe these positive parental behaviors and imitate this type of behavior as well as learn how to solve problems using positive, rather than negative strategies. Parents using consistent, positive parenting techniques may be more likely to nurture the development of protective factors, such as attachment. Parents with less stress may also interact with their child more frequently, thus allowing the child more opportunities to exercise initiative and self-control behaviors. These positive behaviors elicit more positive responses from parents and others with whom they interact, thus reinforcing children's use of positive behaviors.

It is interesting that the results of the current study found that parent-child dysfunctional interactions were a significant mediator, but not parental distress. The Parent-Child Dysfunctional Interaction subscale evaluated the parents' perception of their interactions with their child and measured negative perceptions that a parent has about their child, such as (a) the child does not meet their expectations or (b) the interactions with the child are not reinforcing. Given that this subscale assessed the parents' perceptions of interactions with their child, it may be a more direct measure of their actual behavior with their child compared to the parental distress subscale. For example, if parents rated their perceptions of interactions with their child as negative or endorsed items that suggest negative parental behaviors toward their child, this may be a better predictor of their actual behavior. Negative interactions may have a greater impact on their child's behavior than parents' perceptions of child-rearing competence, social support, and stresses associated with the restrictions placed on other life roles.

It may also be the case that the Parental Distress subscale is more closely related to parental emotional health (Haskett, Ahern, Ward, \& Allaire, 2006). In Haskett and colleagues' (2006) analysis of the psychometric properties of the PSF-SF and its subscales, they found that the Parental Distress subscale (which they referred to as Personal Distress in their article), was strongly related to parents' emotional health. Emotional health was measured by the Symptom Checklist-90-Revised, which measures a broad range of psychological problems. The analysis also revealed that the Parent-Child Dysfunctional Interaction subscale, combined with some items from the Difficult Child subscale, created a subscale they referred to as Child Rearing Stress. This was strongly related to children's behavior problems and parental behavior. The results of Haskett and colleagues' (2006) study suggest that these subscales measure two different types of stress. Considering this in regards to the current study, the Parent-Child Dysfunctional Interaction subscale may be measuring actual parent behavior more closely. Parental Distress may not play a mediating role if it is experienced in isolation and without negative parenting behaviors.

Furthermore, the Parental Distress subscale has been found to be more strongly associated with negative life events than the Parent-Child Dysfunctional Interaction (Whiteside-Mansell et al., 2007). This suggests that this subscale may be less related to only stress as it relates to parenting and may be more encompassing of other life stressors. Additionally, the study found support for the decomposition of the Parent-Child Dysfunctional Interaction subscale into two additional subscales, Dyadic Interaction and Perception of Child. The Dyadic Interaction score was found to be associated with aggressive child behavior, therefore providing support that the Parent-Child Dysfunctional Interaction subscale as a whole may be more strongly associated with child behavior.

\subsection{Limitations and Future Directions}

While a comparison group was included in the current study, an important limitation of the current study was the absence of a control group and lack of random assignment. As with other studies that examine children across time, it is not possible to rule out maturational effects when examining changes in children's behavior. As children grow during the preschool period, research has suggested that core executive function components develop (Garon, Bryson, \& Smith, 2008). Children improving in these areas would likely demonstrate improvements in behaviors that parents or providers rated as problematic. It would be important to investigate why and how children and parents improved, even if they are not participating in an intervention. It is important to ensure that treatment improves children's behavior beyond the natural maturational effects they are experiencing in order to best serve children and utilize resources effectively.

It is unknown whether parents and children who chose to participate in the ECMHC program were different from parents and children that had similar behavior difficulties who did not access services, and whether families in the comparison group were different from families who chose not to. Although this is the case, the CCEP 
program had the potential to impact participants in the ECMHC group through the services that it provided (e.g., education, skill building, consultant support) therefore differentiating them from the comparison group, despite its limitations and lack of control for maturational effects.

One of the advantages of the type of consultation approach used in the current study is its flexibility in service delivery to meet the needs of each individual family. Given that this type of ECMHC program was not a manualized consultation approach it is difficult to know which services were implemented and which were effective. These limitations are consistent with other ECMHC studies in the literature (Gilliam, 2007; Perry et al., 2010).

\section{Conclusions}

High parenting stress levels have been associated with more behavior problems and fewer protective factors in children (e.g., Anthony et al., 2005; Bender \& Carlson, 2013) and the current study further supports this link. Given this, it is important to target parent behavior in treatment by helping parents improve their own parenting behavior and interactions with their child. While many other factors have also been found to be associated with behavior problems (e.g., violent communities, low SES), parent-child interactions may be one factor that is more malleable and responsive to change in intervention services, when compared to other factors such as a the type of community one resides in or the amount of financial resources a family has available (Kazdin \& Whitley, 2003).

The findings of the current study, as well as previous studies, have suggested parents' negative perceptions of the child and the child's behavior also mediate children's outcomes following treatment. Therefore, in addition to targeting parent-child interactions in intervention, it would be important to help parents alter their negative perceptions of the child through cognitive techniques such as reframing. Evaluating negative perceptions of the child may also serve as a preventative measure, given that this may be a precursor to negative interactions with the child. Additionally, parenting stress literature has suggested that an educational component in treatment for children's behavior problems helps reduce stress experienced by parents. Education about child development, the nature of behavior problems, reasonable expectations for a young child, and becoming more sensitive to a child's needs, may also help reduce negative perceptions of the child and the child's behavior.

It is important to continue investigating the differences between parent-child dysfunctional interactions and parental distress. While both of these constructs have been found to be predictors of children's behavioral problems, only parent-child dysfunctional interactions were found in this study to mediate children's behavioral outcomes. In addition to the current study's findings, previous research has also suggested that these domains of parenting stress may be different, which may affect children differently and require different types of interventions. Improved understanding of these conceptualizations of parenting stress may help to effectively target the difficulties parents face when parenting. Interventions utilized in ECMHC that accurately target and provide parents with skills and strategies that directly relate to the difficulties they are experiencing are essential to a refinement of services and improved behavioral outcomes.

\section{References}

Abidin, R. R. (1992). The determinants of parenting behavior. Journal of Clinical Child Psychology, 21, 407-412. http://dx.doi.org/10.1207/s15374424jccp2104_12

Abidin, R. R. (1995). Parenting Stress Index (3rd ed.). Odessa, FL: Psychological Assessment Resources.

Anthony, L. G., Anthony, B. J., Glanville, D. N., Naiman, D. Q., Waanders, C., \& Shaffer, S. (2005). The relationships between parenting stress, parenting behavior, and preschoolers' social competence and behavior problems in the classroom. Infant and Child Development, 14, 133-154. http://dx.doi.org/10.1002/icd.385

Barry, T. D., Dunlap, S. T., Cotton, S. J., Lochman, J. E., \& Wells, K. C. (2005). The influence of maternal stress and distress on disruptive behavior problems in boys. Journal of the American Academy of Child and Adolescent Psychiatry, 44, 265-273. http://dx.doi.org/10.1097/00004583-200503000-00011

Bender, S. L., \& Carlson, J. S. (2013). An initial investigation of parenting stress, social-emotional protective factors, and behavior concerns within a Head Start population. Journal of Educational and Developmental Psychology, 3, 113-123. http://dx.doi.org/10.5539/jedp.v3n1p113

Brinkman, T. M., Wigent, C. A., Tomac, R. A., Pham, A. V., \& Carlson, S. J. (2007). Using the Devereux Early Childhood Assessment to identify behavioral risk and protective factors within a Head Start population. Canadian Journal of School Psychology, 22, 136-151. http://dx.doi.org/10.1177/0829573507307612 
Carlson, J. S., Mackrain, M. A., Van Egeren, L. A., Brophy-Herb, H., Kirk, R. H., Marciniak, D., ... Tableman, B. (2012). Implementing a statewide early childhood mental health consultation approach to preventing child care expulsion. Infant Mental Health Journal, 33, 265-273. http://dx.doi.org/10.1002/imhj.21336

Cohen, E., \& Kaufman, R. (2000). Early childhood mental health consultation. Washington, DC: Center for Mental Health Services of the Substance Abuse and Mental Health Services Administration and the Georgetown University Child Development Center. Retrieved from http://www.samhsa.gov

Deater-Deckard, K. (1998). Parenting stress and child adjustment: Some old hypotheses and new questions. $\begin{array}{lllll}\text { Clinical Psychology: } \quad \text { Science } & \text { 314-332. }\end{array}$ http://dx.doi.org/10.1111/j.1468-2850.1998.tb00152.x

Deater-Deckard, K., Dodge, K. A., Bates, J. E., \& Pettit, G. (1998). Multiple risk factors in the development of externalizing behavior problems: Group and individual differences. Development and Psychopathology, 10, 469-493. http://dx.doi.org/10.1017/S0954579498001709

Feinfield, K. A., \& Baker, B. L. (2004). Empirical support for a treatment program for families of young children with externalizing problems. Journal of Clinical Child \& Adolescent Psychology, 33, 182-195. http://dx.doi.org/10.1207/S15374424JCCP3301_17

Fergusson, D. M., Horwood, L. J., \& Ridder, E. M. (2005). Show me the child at seven: The consequences of conduct problems in childhood for psychosocial functioning in adulthood. Journal of Child Psychology and Psychiatry, 46, 837-849. http://dx.doi.org/10.1111/j.1469-7610.2004.00387.x

Garon, N., Bryson, S. E., \& Smith, I. M. (2008). Executive function in preschoolers: A review using an integrative framework. Psychological Bulletin, 134, 31-60. http://dx.doi.org/10.1037/0033-2909.134.1.31

Gilliam, W. S. (2007, May 15). Early Childhood Consultation Partnership: Results of a random-controlled evaluation. Final report and executive summary. Retrieved from http://www.chdi.org/admin/uploads/5468903394946c41768730.pdf

Green, B. L., Everhart, M., Gordon, L., \& Gettman, M. G. (2006). Characteristics of effective mental health consultation in early childhood settings: Multilevel analysis of a national survey. Topics in Early Childhood Special Education, 26, 142-152. https://dx.doi.org/10.1177/02711214060260030201

Haskett, M. E., Ahern, L. S., Ward, C. S., \& Allaire, J. C. (2006). Factor structure and validity of the Parenting Stress Index-Short Form. Journal of Clinical Child \& Adolescent Psychology, 35, 302-312. http://dx.doi.org/10.1207/s15374424jccp3502_14

Hayes, A. F. (2009). Beyond Baron and Kenny: Statistical mediation analysis in the new millennium. Communication Monographs, 76, 408-420. http://dx.doi.org/10.1080/03637750903310360

Hayes, A. F. (2011). SPSS Macro for Mediation. Retrieved from http://www.afhayes.com/spss-sas-and-mplus-macros-and-code.html

Hayes, A. F. (2012). Macro and script rules and frequently asked questions. Retrieved from http://www.afhayes.com/macrofaq.html

Johnston, K., \& Brinamen, C. F. (2012). The consultation relationship-From transactional to transformative: Hypothesizing about the nature of change. Infant Mental Health Journal, 33, 226-233. http://dx.doi.org/10.1002/imhj.21332

Kazdin, A. E., \& Whitley, M. K. (2003). Treatment of parental stress to enhance therapeutic change among children referred for aggressive and antisocial behavior. Journal of Consulting and Clinical Psychology, 71, 504-515. http://dx.doi.org/10.1037/0022-006X.71.3.504

Kirk, R. (2003). Family support: The roles of early years' centres. Children \& Society, 17, 85-99. http://dx.doi.org/10.1002/CHI.741

LeBuffe, P. A., \& Naglieri, J. A. (1999). Devereux Early Childhood Assessment User's Guide. Lewisville, NC: Kaplan Press.

Mistry, R. S., Vandewater, E. A., Huston, A. C., \& McLoyd, V. C. (2002). Economic well-being and children's social adjustment: The role of family process in an ethnically diverse low-income sample. Child Development, 73, 935-951. http://dx.doi.org/10.1111/1467-8624.00448

Patterson, G. R. (1982). Coercive family process. Eugene, OR: Castalia. 
Patterson, G. R., \& Reid, J. B. (1970). Reciprocity and coercion: Two facets of social systems. In C. Neuringer, \& J. Michael (Eds.). Behavior Modification in Clinical Psychology. New York: Appleton-Century-Crofts.

Perry, D. F., Allen, M. D., Brennan, E. M., \& Bradley, J. R. (2010). The evidence base for mental health consultation in early childhood settings: A research synthesis addressing children's behavioral outcomes. Early Education \& Development, 21, 795-824. http://dx.doi.org/10.1080/10409280903475444

Perry, D. F., Dunne, M. C., McFadden, L., \& Campbell, D. (2008). Reducing the risk for preschool expulsion: Mental health consultation for young children with challenging behaviors. Journal of Child \& Family Studies, 17, 44-54. http://dx.doi.org/10.1007/s10826-007-9140-7

Preacher, K. J., \& Hayes, A. F. (2008). Asymptotic and resampling strategies for assessing and comparing indirect effects in multiple mediator models. Behavior Research Methods, 40, 879-891. http://dx.doi.org/10.3758/BRM.40.3.879

Preacher, K. J., Rucker, D. D., \& Hayes, A. F. (2007). Addressing moderated mediation hypotheses: Theory, methods, and prescriptions. Multivariate Behavioral Research, 42, 185-227. http://dx.doi.org/10.1080/00273170701341316

Qi, C. H., \& Kaiser, A. P. (2003). Behavior problems of preschool children from low-income families: A review of the literature. Topics in Early Childhood Special Education, 23, 188-216. http://dx.doi.org/10.1177/02711214030230040201

Raikes, H. A., \& Thompson, R. A. (2005). Efficacy and social support as predictors of parenting stress among families in poverty. Infant Mental Health Journal, 26, 177-190. http://dx.doi.org/10.1002/imhj.20044

Raver, C. C., Jones, S. M., Li-Grining, C., Zhai, F., Metzger, M. W., \& Solomon, B. (2009). Targeting children's behavior problems in preschool classrooms: A cluster-randomized controlled trial. Journal of Consulting and Clinical Psychology, 77, 302-316. http://dx.doi.org/10.1037/a0015302

Reitman, D., Currier, R. O., \& Stickle, T. R. (2002). A critical evaluation of the Parenting Stress Index-Short Form (PSI-SF) in a Head Start population. Journal of Clinical Child and Adolescent Psychology, 31, 384-392. http://dx.doi.org/10.1207/S15374424JCCP3103_10

Rutter, M. (1987). Psychosocial resilience and protective mechanisms. American Journal of Orthopsychiatry, 57, 316-331. http://dx.doi.org/10.1111/j.1939-0025.1987.tb03541.x

Scher, A., \& Sharanbany, R. (2005). Parenting anxiety and stress: Does gender play a part at 3 months of age? The Journal of Genetic Psychology, 166, 203-213. http://dx.doi.org/10.3200/GNTP.166.2.203-214.

Shrout, P. E., \& Bolger, N. (2002). Mediation in experimental and nonexperimental studies: New procedures and recommendations. Psychological Methods, 7, 422-445. http://dx.doi.org/10.1037//1082-989X7.4.422

Upshur, C., Wenz-Gross, M., \& Reed, G. (2009). A pilot study of early childhood mental health consultation for children with behavioral problems in preschool. Early Childhood Research Quarterly, 24, 29-45. http://dx.doi.org/10.1016/j.ecresq.2008.12.002

Van Egeren, L. A., Kirk, R. H., Brophy-Herb, H. E., Carlson, J. S., Tableman, B., \& Bender, S. L. (2011). An interdisciplinary evaluation report of Michigan's Childcare Expulsion Prevention (CCEP) initiative. Retrieved from http://outreach.msu.edu/cerc/research/ccep.aspx

Whiteside-Mansell, L., Ayoub, C., McKelvey, L., Faldowski, R. A., Hart, A., \& Shears, J. (2007). Parenting stress of low-income parents of toddlers and preschoolers: Psychometric properties of a short form of the Parenting Stress Index. Parenting: Science and Practice, 7, 27-56. http://dx.doi.org/10.1080/15295190709336775

\section{Copyrights}

Copyright for this article is retained by the author(s), with first publication rights granted to the journal.

This is an open-access article distributed under the terms and conditions of the Creative Commons Attribution license (http://creativecommons.org/licenses/by/4.0/). 